\title{
Linx
}

Revue des linguistes de l'université Paris X Nanterre

5 | 1994

La négation

\section{La négation comme jugement : une application aux interronégatives}

\section{Claude Muller}

\section{(2) OpenEdition}

1 Journals

Édition électronique

URL : http://journals.openedition.org/linx/1210

DOI : 10.4000/linx.1210

ISSN : 2118-9692

\section{Éditeur}

Presses universitaires de Paris Nanterre

\section{Édition imprimée}

Date de publication : 1 juin 1994

Pagination : 205-221

ISSN : 0246-8743

\section{Référence électronique}

Claude Muller, «La négation comme jugement : une application aux interronégatives », Linx [En ligne], 5 | 1994, mis en ligne le 18 juillet 2012, consulté le 10 décembre 2020. URL : http:// journals.openedition.org/linx/1210; DOI : https://doi.org/10.4000/linx.1210

Ce document a été généré automatiquement le 10 décembre 2020.

Département de Sciences du langage, Université Paris Ouest 


\title{
La négation comme jugement : une application aux interronégatives
}

\author{
Claude Muller
}

J'ai exposé récemment (Muller, 1992) le point de vue selon lequel la négation exprime un jugement sur un énoncé (en l'occurrence, un jugement de rejet - l'usage du terme rejet pour qualifier l'emploi principal des négations a été proposé par P. Attal, 1979), comparable en cela non à l'assertion affirmative, mais à la confirmation d'un énoncé vu ou présenté comme antérieur. On sait qu'il est difficile de définir un « acte » spécifique de négation (cf. par exemple Moeschler, 19911ㅗ. Dans cette analyse, la négation retrouve son unité - un jugement peut être asserté, mais aussi rapporté («Il a dit que non») ou enchâssé. Puisqu'il s'agit d'un jugement d'inadéquation de l'énoncé, il est normal que l'usage qui en soit fait soit surtout de rejeter un énoncé, mais l'acte alors accompli - le fait d'assumer ce jugement de rejet, pour le locuteur - est une forme d'assertion. Enfin, il ne s'agit pas de nier la distinction entre la négation "polémique» et la négation « descriptive » : dans l'usage polémique, le locuteur n'assume que l'assertion du jugement de négation, pas celle du reste de l'énoncé. La théorie de la polyphonie permet cette distinction d'une façon éclairante: le locuteur est responsable de la négation, la proposition sur laquelle elle porte est présentée comme relevant d'un autre énonciateur. Par contre, dans la négation descriptive, le locuteur utilise la négation comme un prédicat parmi d'autres : il est également l'énonciateur de l'argument de la négation. D'où l'aspect concerté de la phrase sur laquelle porte une négation descriptive: le positif est évidemment choisi en fonction de la négation, pour «faire sens » avec lui².

On remarquera qu'ainsi se trouve éliminée l'asymétrie signalée par Bruno Callebaut entre l'analyse de la négation polémique, définie comme telle grâce à son contexte plutôt que par son contenu, et l'analyse de la négation descriptive, définie en principe par son analyse sémantique interne. Dans l'analyse que je propose, le critère est celui de la distinction ou non des énonciateurs, ce qui est un critère interne, dont la détermination demande évidemment la prise en considération du contexte. Le caractère très limité des possibilités de négation descriptive est également facile à comprendre : une condition nécessaire à son emploi est que la combinaison de la négation et de la chaîne prédicative 
sur laquelle elle porte soit sémantiquement intéressant du point de vue descriptif, puisque la négation est choisie pour cela. Et rien n'empêche, avec la même chaîne prédicative, d'avoir affaire à une négation polémique si le locuteur se démarque de l'énoncé positif, soit parce qu'il est de quelqu'un d'autre, soit parce qu'il s'agit d'un point de vue qu'il ne partage pas.

II. Je voudrais maintenant examiner une possible application de ce point de vue aux interronégatives. Ce domaine est maintenant bien connu, grâce entre autres aux travaux d'Andrée Borillo et de Bruno Callebaut. Je crois que tout le monde s'accorde à y voir au moins deux usages distincts de la négation :

- un usage que B. Callebaut appelle la NEGATION VRAIE, dans lequel la négation se combine avec ses arguments à la manière de la négation des phrases assertées, et qui correspond à l'usage défini comme CONFIRM-NON par A. Borillo, 1979.

- et un usage appelé NEGATION ARGUMENTATIVE, caractérisé par la possibilité de supprimer la négation sans changer le sens, et plutôt CoNFIRM-OUi (Callebaut, 1991, p 94).

En dehors de ces deux types, il y a encore la question alternative (question ouI-Non) qui est selon A. Borillo la source syntaxique des questions positives ou négatives, ce qui n'est pas admis par tout le monde (cf. de Cornulier).

Je laisserai de côté la question alternative, qui me paraît spécialisée dans l'insistance (quelle que soit sa source dérivationnelle), avec le contexte fréquent d'une question identique antérieure laissée sans réponse (opinion de Sennekamp et Callebaut).

Un exemple de chaque emploi, sans faire intervenir les critères syntaxiques comme l'inversion interrogative ou la présence ou l'absence de mots associés à la négation - pour rester dans les cas les plus simples :

(1) Tu ne te sens pas bien?

Ici, la réponse attendue - c'est-à-dire, jugée plausible, sans être souhaitée évidemment est une réponse d'accord négatif :

- En effet, je ne me sens pas très bien.

On a alors affaire à l'interronégative à négation dite vraie.

Autre cas :

(2)· J'aimerais te trouver des excuses, mais est-ce que tu ne te sens pas responsable de ce qui s'est passé?

Dans ce cas, la négation est plutôt du type argumentatif, sans être une question purement rhétorique, ce que font bien remarquer Anscombre et Ducrot pour ce type d'exemples.

Dans ce qui suit, je vais réexaminer quelques cas caractéristiques d'interronégatives, et voir si l'analyse que je propose de la négation permet d'en rendre compte.

III. En réfléchissant sur les interronégatives, j'ai été amené pour les comprendre à faire une distinction entre deux plans qui correspondent grosso modo au présupposé et au posé de l'assertion, mais pour lesquels il faut une caractérisation spécifique, puisque sauf question rhétorique, rien n'est acquis du contenu propositionnel dans la question. Le problème est identique à celui que posent les phrases négatives dans lesquelles le positif semble présupposé, sans l'être vraiment puisque la négation détruit la croyance dans le 
positif : c'est ce que Givon appelle les présupposés pragmatiques, et ça correspond à un des points de vue énonciatifs de l'analyse polyphonique de Ducrot.

Je répartirai les questions totales, quelles qu'elles soient, en deux catégories préliminaires :

- questions avec ou questions sans arrière-plan ${ }^{3}$

L'arrière-plan, c'est l'opinion préalable de la part du locuteur sur la proposition (je préfère le terme vague d'opinion à l'expression "assertion préalable » employée par Anscombre et Ducrot). C'est plus ou moins le présupposé, non pas logique, mais pragmatique puisqu'il ne s'agit pas nécessairement d'une proposition à la vérité établie. Il me semble en effet que l'attitude propositionnelle du locuteur laisse percevoir dans la question son opinion préalable - s'il en a eu une - sur la vérité de la proposition (qu'il convient de distinguer de la réponse qu'il juge probable).

A l'arrière-plan s'oppose le premier plan: j'y mettrai et la question en tant qu'acte d'énonciation, et la motivation directe de la question : tout se passe comme si, précédant immédiatement son énonciation, le locuteur prenait soudain conscience d'un certain état de choses, pouvant remettre en cause son opinion d'arrière-plan si il en avait une; il me semble que beaucoup de questions sont ainsi motivées. Par exemple, si le locuteur a déjà une opinion d'arrière-plan, le premier plan sera constitué d'indices - comportement de l'interlocuteur ou faits quelconques - qui l'amèneront à douter de son opinion première. Les éléments linguistiques du premier plan seront alors dépendants de la stratégie adoptée, car il pourra y en avoir deux : dans notre exemple, ça pourrait être une question sur le bien-fondé de la croyance d'arrière-plan, ce qui se traduira par la mise en forme interrogative de cet arrière-plan. Dans ce sens, l'interrogation de premier plan est orientée vers une possible réponse négative qui rejettera cet énoncé.

L'autre stratégie, motivée de la même façon, va privilégier les indices de premier plan : le locuteur construit un énoncé conforme à ces indices (donc opposé à sa croyance d'arrière-plan), en anticipant sur ce qu'il croit être la bonne réponse. Alors, l'interrogation est orientée vers une réponse de confirmation.

Un exemple, aussi simple que possible : je suppose que je crois me diriger vers la gare. Pourtant, quelque chose dans le décor me fait penser que je puis me tromper. L'arrièreplan est donc quelque chose comme : «je suis dans la bonne direction». Je peux alors, en demandant mon chemin, soit mettre en question cette croyance d'arrière-plan, en disant quelque chose comme :

(3) Je voudrais aller à la gare, mais je ne reconnais plus mon chemin. Suis-je dans la bonne direction?

soit, supposant que je me suis trompé, anticiper sur la réponse de mon interlocuteur, en disant :

(4) Je voudrais aller à la gare, mais je ne reconnais plus mon chemin : est-ce que je ne suis pas dans la bonne direction?

Il ne s'agit pas de questions à orientations différentes : toutes deux sont plutôt conçues en prévision d'une réponse négative. C'est la stratégie suivie qui diffère.

Autre exemple, évidemment imaginaire : un journal à sensation croit déceler dans les propos du pape un soupçon de scepticisme à l'égard des croyances de la religion chrétienne. Le présupposé d'arrière-plan est quelque chose comme : «si on est pape, on croit en Dieu ». Le gros titre du journal pourrait être, soit :

(5) Le pape croit-il (vraiment) en Dieu ? 
ce qui serait une mise en doute de l'arrière-plan, soit :

(6) Le pape ne croit (croirait)-il pas en Dieu?

ce qui est une forme d'anticipation de la réponse suggérée, donc une formulation qui intègre la motivation de premier plan. Les deux questions se différencient par les marqueurs linguistiques qui les accompagnent: les adverbes vraiment, bien, dans le premier cas ; le conditionnel, ou une paraphrase en peut-être dans le second cas ${ }^{4}$.

IV. On remarquera que ces questions restent de vraies questions. Ce n'est pas toujours le cas, et la prise en compte de la polyphonie permet de l'expliquer. Restons dans la même situation : un arrière-plan consistant en une proposition affirmative, le premier plan peut contenir une motivation qui n'est pas cette fois la remise en cause par le locuteur luimême de cette croyance, mais sa mise en cause par l'interlocuteur. Bien évidemment, c'est le locuteur qui peut "mettre en scène" cette mise en cause, il n'est pas indispensable qu'elle soit réelle. Ce faisant, le locuteur ne partage pas lui-même cette remise en cause : il l'attribue à son interlocuteur, et sa question met en demeure celui-ci de rejeter sa mise en question, donc de confirmer l'arrière-plan. On aura donc affaire cette fois à une question à orientation affirmative. Un exemple :

(7) Est-ce que je ne viens pas de t'aider?

Je présuppose évidemment que «je viens de t'aider »; mon interlocuteur, à mon avis, se comporte comme si ça n'était pas le cas. On se trouve donc, répartition des rôles mise à part, dans la situation déjà vue d'une remise en question d'un énoncé positif en arrièreplan. Cependant la question, qui est formellement du type de ce qu'on appelle une négation d'anticipation, n'appelle pas une réponse négative, mais un rejet de celle-ci, et une confirmation de l'énoncé d'arrière-plan, jamais mis en doute par le locuteur. On voit ainsi que la possibilité, pour le locuteur, de se dissocier nettement en tant qu'énonciateur du point de vue du premier plan, permet à la même structure d'avoir une orientation inverse : la question négative répartit les rôles: le locuteur adopte le point de vue de l'énoncé positif sous-jacent, et attribue à l'interlocuteur celui de son rejet, et la question qui a la forme de l'énoncé négatif attribué à l'interlocuteur, faisant mine d'anticiper sur sa réponse, vise à lui faire constater d'évidence le caractère insoutenable d'une assertion négative de ce type. On n'est plus dans la recherche de l'énoncé adéquat, mais dans la simulation de ce que croit l'interlocuteur, qu'il s'agit de convaincre d'incohérence. Cette utilisation argumentative et rhétorique ne remet donc pas en cause le schéma initialement exposé, elle en montre une utilisation dialogale particulière.

Récapitulons : avec un énoncé positif en arrière-plan, trois types de question au moins sont envisageables: une interropositive de mise en doute de l'arrière-plan; une interronégative qui anticipe sur une réponse négative, toutes deux orientées vers une réponse négative; enfin, une interronégative qui «met en scène » l'interlocuteur, mais avec l'intention d'obtenir le rejet de son point de vue, donc une réponse affirmative confortant l'arrière-plan positif.

Peut-être est-il également envisageable qu'il y ait des interropositives qui «mettent en scène » avec la même valeur une mise en doute attribuée à l'interlocuteur d'un AR-PL positif que le locuteur, quant à lui, tient toujours pour vrai :

Tu as bien dit il y a moins de $5 m n$ que l'incident était clos?

afin d'obtenir une confirmation positive; mais de tels énoncés me semblent généralement moins plausibles. 
Quel usage est-il fait de la négation dans les deux cas (ex. (4) et (7) ) où on en trouve une ? Il me semble que la négation dite "vraie » dans la terminologie de B. Callebaut, de type CONFIRM-NON et la négation dite " argumentative » de type CONFIRM-OUI - du moins dans le type qu'on vient d'examiner - sont très proches, comme on a essayé de le montrer : dans les deux cas, il y a anticipation d'une réponse possible de l'interlocuteur ; simplement, dans la négation « vraie» (ex. (4)), il y a également un doute du locuteur sur la croyance d'arrière-plan (un "déficit cognitif » selon Callebaut) si bien que le locuteur est prêt à admettre l'opinion - positive ou négative - du questionné. Dans l'autre cas, (ex. (7) ) la croyance négative suggérée comme étant le point de vue de l'interlocuteur est proposée comme insoutenable, et méritant d'être rejetée, et le locuteur fait comprendre qu'il ne la partage pas, et qu'il ne l'acceptera pas sans explications.

On connaît le côté paradoxal de ce type de questions, souligné par A. Borillo et B. Callebaut, que je cite : «d'où est-ce qu'une négation tire ce pouvoir étrange d'orienter une question - ou plutôt une réponse - vers une valeur de vérité qui lui est en plus opposée ?! Et, en fin de compte, que faire d'une négation argumentative considérée comme exclusivement liée à l'interrogation, dans une théorie globale de la négation? » (Callebaut, p. 94).

Bruno Callebaut se range à l'explication d'Andrée Borillo, donnée dans son article de 1979 : cet usage relève de la stratégie dialogale : « Lorsqu'il (= le locuteur) dit “non $(P)$ ?", en même temps qu'il formule l'éventualité d'une assertion négative de la part de son interlocuteur, le locuteur signale qu'il n'est pas prêt à la recevoir telle quelle sans explication, argumentation ou justification. La manière interrogative qu'il a d'évoquer cette éventualité indique en quelque sorte qu'elle fait problème pour lui. Bien sûr, la raison en est simple, c'est qu'il croit, lui, à l'assertion de la valeur inverse, la valeur positive de la proposition. » (A. Borillo, p. 38).

En simplifiant, on peut dire que la négation est ici un jugement attribué à l'interlocuteur, par anticipation, et à lui seul, puisque le locuteur s'en démarque. L'interlocuteur est donc mis dans l'obligation, ou de se déjuger, ou de se justifier sérieusement. Il n'y a rien là qui aille à l'encontre de l'analyse proposée pour la négation dans les phrases assertives : au contraire, puisqu'il y a opposition tranchée entre l'énonciateur de la négation et celui du positif sous-jacent, on retrouve un des aspects de la négation polémique : la distinction maintenue aussi nette que possible entre la négation et l'énoncé affirmatif, qui ne relèvent pas du même énonciateur.

Cette analyse polyphonique de la question CONFIRM-ou de type rhétorique a l'avantage de pouvoir expliquer pourquoi, dans ce type de question négative, la négation a tendance à s'amalgamer avec l'interrogation, formant assez facilement des locutions comme n'est-ce pas que... Andrée Borillo se demandait s'il n'y avait pas là un phénomène de variation de portée : cela me paraît exclu, je croirais plutôt que, comme la négation polémique, ou à plus forte raison la négation métalinguistique, qui se traduit souvent par une position externe de la négation, pour mieux séparer les points de vue soutenus par des énonciateurs différents, il y a ici une rupture marquée entre d'une part, la question et son argument qui est donc le jugement de négation prêté à l'interlocuteur, l'ensemble formant la mise en scène dialogale évoquée par Andrée Borillo, et la proposition ellemême, que le locuteur croit vraie. Ainsi, la question et son argument la négation forment un opérateur fermé qui va porter globalement sur la proposition ${ }^{5}$. La portée est la même que dans les autres cas d'interronégatives, mais il se fait dans ce cas, entre la question et son argument la négation, une sorte d'amalgame local ressemblant à l'association négative entre la négation et certains quantifieurs. La proposition n'est pas hors de la 
portée de la négation, comme l'a écrit Bruno Callebaut (du moins à propos des termes sensibles à la portée, p. ex. p. 60) mais évidemment cela y ressemble fort puisque l'amalgame questionXnégation a une portée doublement négative (c'est en gros la mise en doute d'un jugement négatif) donc affirmative.

V. Cependant, il y a d'autres situations à prendre en considération: j'examinerai rapidement celles - symétriques des situations déjà examinées - où l'on peut admettre un arrière-plan négatif, en suivant l'ordre des cas à arrière-plan positif :

- l'interrogation est une mise en doute de l'arrière-plan négatif : elle contient donc une négation, partie intégrante de la proposition:

(8) Tu n'as (vraiment) vu personne?

Tu ne m'as jamais menti?

L'orientation est positive : la réponse jugée plausible/probable serait par exemple : oui, je t'ai déjà menti.

- l'interrogation est une anticipation de la réponse affirmative qu'on pressent, opposée à l'arrière-plan (l'inverse donc de la négation de confirmation) :

(9) Tu en as parlé à quelqu'un?

Tu m'as peut-être menti?

Dans les deux cas, la stratégie du locuteur est la même et présente comme la plus plausible une réponse positive.

On peut ici aussi imaginer un journal présenter en titre, soit :

(10) La contamination des hémophiles: le ministre de la santé n'était-il (vraiment) pas au courant?

qui est une mise en doute de la proposition négative d'arrière-plan, soit :

...le ministre de la santé était-il au courant ?

qui serait une question affirmative par anticipation.

Enfin, la même stratégie de mise en scène d'une opinion prêtée à l'interlocuteur peut être utilisée à partir d'un arrière-plan négatif. Le locuteur présentera alors sous forme de question un énoncé qui rejettera son propre point de vue, et qu'il attribuera au locuteur, ainsi également mis en demeure de s'expliquer. On aura alors affaire à une interropositive rhétorique, avec une orientation de confirmation de l'arrière-plan, donc négative : 6

(11) Est-ce que Paul a jamais levéle petit doigt pour t'aider?

Est-ce que je t'ai déjà refusé quoi que ce soit?

Est-ce que tu me prends pour un idiot?

Dans tout cela, la négation se comporte comme un jugement, utilisé parmi d'autres éléments dans des actes illocutoires complexes. Rien ne permet de dire qu'il y ait une négation particulière, argumentative par exemple : l'argumentativité utilise la négation comme elle le fait des autres éléments propositionnels. La différence essentielle entre les questions confirm-non et les questions rhétoriques confirm-oui est affaire de polyphonie, pas de la négation elle-même, on espère l'avoir montré.

VI. Il y a cependant un type de question négative confirm-oui qui me paraît abusivement confondu avec celui dont on vient de parler en termes de stratégie dialogale et de mise en scène. Il s'agit de ces questions sans arrière-plan particulier, non liées nécessairement au dialogue, et qui sont souvent accompagnées de l'adverbe « par hasard» :

(12) Vous n'êtes pas M. Martin, par hasard?

Est-ce que je n'aurais pas par hasard oublié mes clefs? 
Ça nous conduit à examiner le cas de questions sans arrière-plan. On peut en effet imaginer que certaines des questions réelles de la vie courante sont immédiatement motivées, c'est-à-dire qu'elles consistent en une construction simultanée de la proposition et de la question. En ce sens, le premier plan contient aussi des propositions, et pas seulement des jugements. Ainsi, voyant Marie après quelques temps, pourrais-je dire (si je la connais assez pour n'être pas impoli) :

(13) Tiens, tu es enceinte?

sans avoir jamais présupposé qu'elle ne l'était pas, du moins sans avoir jamais eu à l'esprit la proposition négative correspondante. On le sait, on ne passe pas en revue toutes les circonstances négativement vraies: Wittgenstein en a fait la remarque : "J'admets que la maison dans laquelle j'écris ne va pas s'écrouler dans la prochaine demi-heure. Quand est-ce que je l'admets : tout le temps ?... ». Le premier plan construit directement la proposition et pose la question pour confirmation. L'interropositive est alors une anticipation de la réponse probable. Mais il existe aussi des interronégatives sans arrière-plan: des questions comme :

(14) Ça ne va pas?

Tu ne te sens pas bien?

sont directement motivées par la situation, sans énoncé antérieur apparent, et comme les interropositives qu'on vient de voir, elles anticipent sur la réponse jugée la plus probable.

En y regardant de plus près on remarquera que ces questions sans arrière-plan sont plus généralement affirmatives : pour qu'une question négative du type de (14) soit naturelle, il faut que l'état normal des choses soit une propriété exprimée sous forme affirmative (quelque chose comme : normalement, ça va; normalement, on se sent bien; alors qu'on ne peut avoir l'opinion suivante : normalement, une femme est enceinte).

Pourtant, les interronégatives de type "par hasard» semblent bien être de la catégorie des interropositives sans arrière-plan du type de (13), qui est paraphrasable par (15) :

(15) Tu n'es pas enceinte, par hasard?

puisqu'elles ne laissent apparaitre aucune opinion préalable du locuteur et qu'elles sont, comme les interropositives du même type, orientées vers une possible confirmation affirmative.

Ce type de question me semble véritablement faire problème : il ne peut vraiment pas se rattacher au type rhétorique vu ci-dessus, qui suppose un arrière-plan positif pour le locuteur, et une répartition des rôles d'énonciateurs. Dans ce type de questions, la proposition est véritablement introduite comme " nouvelle », directement motivée. Il n'y a pas plus d'arrière-plan que dans les questions de type "pourquoi pas", qui ne présument aucun rejet dont on demanderait la cause :

(16) Pourquoi ne pas venir déjeuner un prochain jour?

est une invitation, pas une demande d'explication sur un rejet. Deux exemples réels :

(17) Pourquoi ne pas songer à la Jordanie pour être cette patrie géographique du peuple palestinien? (Le Monde, 30.7.82)

(18) Pourquoi pas aussi ne pas le faire sans que le législateur n'intervienne? (Le Monde, 29.7.82)

dont le dernier montre la dissociation du pourquoi pas et de la proposition, elle-même négative, mais avec deux négations qui jouent le même rôle, puisque le sens est :

Il faut le faire sans que le législateur n'intervienne.

VII. Il me semble nécessaire d'expliquer de la même façon la négation des questions " par hasard » et des questions en «pourquoi pas ». Dans les deux cas, il y a introduction d'une 
proposition présentée comme nouvelle, du domaine de la croyance ou du domaine du faire.

On peut imaginer en gros trois types d'explications : dans l'un, la négation porterait sur la proposition, et la question mettrait en doute la négation, rétablissant ainsi la polarité positive et orientant la réponse vers l'affirmation. Il me semble exclu d'accepter tel quel ce type d'explication, puisqu'il y a une différence nette dans la signification et les conditions d'emploi de la question "par hasard» et de ce type d'interronégative qui serait la mise en doute d'un arrière-plan négatif (ex. (8) ci-dessus). Ce type de question (mise en doute d'un AR-PL négatif) suppose évidemment un arrière-plan, et la négation y reste compatible avec l'association négative :

(19) Tu n'as (vraiment) besoin de rien?

(rappel, dans un contexte où l'interlocuteur a déjà dit qu'il n'avait besoin de rien)

s'oppose ainsi à la question sans arrière-plan :

(20) Tu n'as pas besoin de quelque chose, par hasard?

(je viens de me demander, au vu du comportement ou de l'attente de l'interlocuteur, si celui-ci avait besoin de quelque chose)

De même, la question pourquoi portant sur une phrase négative serait une demande d'explication sur un état de fait négatif présupposé (présupposition qui explique l'acceptabilité de la semi-négation jamais :) :

(21) Pourquoi ne lui en parles-tu pas (jamais) quand tu le rencontres?

- Parce que ça m'ennuie de lui demander service.

vs. :

(22) Pourquoi ne lui en parles-tu pas ( ${ }^{*}$ jamais) ?

- C'est une excellente suggestion.

Faut-il alors, deuxième explication, supposer une négation utilisée uniquement en combinaison avec la question, pour introduire une proposition nouvelle présentée comme probable? Il y a des indices dans ce sens. Outre l'aspect sémantique déjà signalé, le fait que les contraintes d'argument ne sont plus celles de la négation: elles sont propres à la combinaison des deux modalités : on peut dire :

(23) Est-ce qu'on ne s'est pas déjà rencontré, par hasard?

à comparer avec: *On ne s'est pas déjà rencontré.

Contre cette explication, qui supposerait qu'aucun lien ne subsiste entre la négation et la proposition construite comme argument, il y a cependant le phénomène signalé par Andrée Borillo (et en (14) ci-dessus) : que certaines questions négatives s'interprètent mal ainsi. Ajoutons, dans notre système d'interprétation, qu'il s'agit aussi de questions sans arrière-plan. Ce sont des questions comme (14) ou (24) :

(24) Est-ce que ça ne va pas?

Est-ce que tu n'as pas compris?

qui sont plutôt orientées vers une réponse d'accord négatif, et difficiles à interpréter autrement. On a signalé ce qui paraît justifier leur occurrence : l'état normal des choses est que ces propositions sont considérées comme admises, allant de soi, plus naturelles en tout cas que leur équivalent négatif. Il est normal que l'interlocuteur ait compris, et une question « par hasard » est vraiment bizarre :

(25) *Est-ce que ça ne va pas, par hasard? (sens : ça va, et j'en suis surpris)

*Est-ce que, par hasard, tu n'as pas compris? (sens : tu as compris, et c'est vraiment surprenant) 
On en déduit donc que l'interprétation qui nous intrigue n'a pas cette propriété. Il s'agit de propositions qui, dans ce qui va de soi, ne sont pas admises comme positives : il ne va pas de soi, par exemple, qu'une jeune femme est enceinte, ou que moi, locuteur, j'oublie mes clés. Autrement dit, la proposition se construit non pas à partir de rien, mais à partir d'une opinion commune sur l'état probable des choses lorsque nous n'en avons aucune information préalable : les interronégatives " par hasard », sont en contradiction avec cet état probable des choses. Ne faut-il pas alors supposer que, en formant cette proposition comme nouvelle, on fasse comme si on rejetait ou on mettait en question un «ordre normal des choses » considéré comme établi ? Cela expliquerait l'effet de surprise associé ici à la négation, et la compatibilité avec l'adverbe "par hasard». Qu'est-ce qui justifie son occurrence ${ }^{7}$ ? Sous réserve d'un examen approfondi, je dirais que c'est un adverbe qui exprime l'incertitude du locuteur sur la validité de la proposition qu'il introduit : quelque chose : j'avance cette idée par hasard, sans certitude.

VIII. Compte tenu du sens de ce type de question - en fait, l'émergence d'une proposition non avérée, donc surprenante et modifiant l'état « normal » des choses, j'y verrais - avec précautions - le mécanisme suivant: le locuteur crée un arrière-plan fictif (c'est-à-dire sans antériorité chronologique réelle) négatif, justifié par l'état normal des choses, en vertu du principe qu'une proposition nouvelle, donc non avérée et qui - chose importante - ne va pas de soi - peut être considérée comme une proposition niée aussi longtemps que non admise, et le premier plan consiste à mettre en doute ce jugement négatif d'arrièreplan, donc à aller vers une assertion de la proposition affirmative, dont l'interronégative représente une première étape ${ }^{8}$. L'avantage sur l'interropositive est certain: d'une part, la polarité résultante est affirmative ; d'autre part, il n'y a pas de confusion possible avec l'interropositive qui met en doute la proposition, donc est orientée négativement. Enfin, le dispositif qui fait contraster arrière-plan et premier plan induit un effet de contraste sémantiquement exploitable, pour souligner le caractère sinon surprenant, du moins nouveau, de la proposition sur laquelle on s'interroge.

On peut schématiser ainsi les deux types de question à AR-PL négatif :

AR-PL négatif antérieur :

- L'AR-PL est présupposé pragmatiquement ;

- L'AR-PL est « entretenu » dans la conscience des interlocuteurs.

AR-PL négatif sans antériorité :

- L'AR-PL n'est pas présupposé pragmatiquement : mais il est décidable sous la forme d'une opinion basée sur la connaissance du monde : normalement, jusqu'à plus ample informé, non $P$.

- L'AR-PL ne préexiste pas à la question dans la conscience des interlocuteurs.

De même, la question pourquoi pas introduit la suggestion d'une action comme état de choses non encore accompli, et dont on demande la cause. Cela autorise une réponse littérale en parce que seulement si l'état des choses négatif a déjà été réellement envisagé, donc est présupposé pragmatiquement et entretenu dans la conscience des interlocuteurs. Par contre, s'il s'agit d'un état de choses entièrement nouveau, envisageable mais non accompli, ce qui justifie par le même mécanisme la forme négative, c'est le contraste entre la proposition négative actuelle et sa possible réalisation future qui prime : l'énoncé est alors suggestion d'agir. En somme, la même différence de nature de l'AR-PL négatif rend compte du contraste entre la question sur la cause d'un non-agir, et la suggestion d'une action à faire. 
IX. Que conclure de ceci sur l'analyse de la négation? Il est clair qu'ici, la négation joue un rôle essentiel dans le complexe illocutoire qui donne à l'interronégative son orientation particulière. Il est clair aussi, je pense, qu'une analyse de la négation en tant qu'acte illocutoire est exclue ici, et qu'il est également impossible d'en faire un simple dérivé locutoire portant sur le contenu propositionnel. En termes de jugement - moins contraignants puisqu'échappant aux définitions de l'acte illocutoire, il faut tout de même un support-l'énonciateur qui assume ce jugement. Or comment supposer un énonciateur antérieur pour une proposition non encore avérée, donc non envisagée jusque là, et par conséquent non susceptible d'être rejetée? On retrouve le paradoxe de Wittgenstein s'interrogeant sur le degré de réalité des propositions négatives vraies qu'on peut difficilement considérer comme présupposées. En gros: y a-t-il un point de vue présupposé sous-jacent qui serait "l'ordre normal des choses jusqu'à plus ample informé ", utilisable dans la construction d'une proposition nouvelle comme mise en doute de la proposition négative ? Cela paraîtra peu vraisemblable si on imagine que l'on ait quelque part conscience des propositions négatives vraies, qui ne correspondent à aucune perception de la réalité. Par contre, un présupposé global comme: Aussi longtemps qu'une proposition non naturellement vraie n'est pas établie, elle est à considérer comme fausse, permettrait de comprendre ce qui se passe. La proposition nouvelle se heurte lorsqu'elle se forme à ce présupposé global. Dès lors, une stratégie de mise en doute de ce présupposé peut se déployer et présenter la proposition affirmative comme plausible sans être établie. Cela donne encore un support sémantique et cognitif à la négation, comme jugement structurant notre reconnaissance du monde.

Les conditions d'occurrence de ces constructions vont dans ce sens d'une mise en doute d'une opinion préalable négative, qui tient compte du contenu propositionnel puisque celui-ci doit être introduit comme un fait nouveau dans la connaissance du monde des interlocuteurs. Pour ce faire, il est donc nécessaire que ce fait nouveau ne soit pas considéré comme préexistant, ou normalement réalisé. L'usage de la négation dans ce cas paraît tout à fait intéressant et singulier : s'il y a jugement, celui-ci n'est pas, comme dans l'usage habituel, jugement sur un contenu propositionnel préalable. Il s'agirait plutôt de l'utilisation «à découvert » d'un usage implicite, qui consiste à classer comme fausse toute proposition qui n'est pas naturellement vraie aussi longtemps qu'elle n'est pas établie. Le "naturellement établi » suit ici aussi les critères de satisfaction déjà mis en évidence par Givon, et d'autres, dans le classement des prédicats. Ainsi, on opposera :

Je me demande si Paul n'est pas malade

et :

\section{?? ?Je me demande si Paul n'est pas en bonne santé}

question sémantiquement déviante sans présupposé antérieur de maladie, puisqu'il est normal, dans notre vision du monde, que la personne dont on parle soit en bonne santé lorsqu'on n'a aucune information à ce sujet.

Une remarque sur la syntaxe: la négation ne se combine pas avec le contenu propositionnel (on doit admettre ici aussi un amalgame de la négation et de la question). Cette propriété semble aller contre une analyse en termes d'arrière-plan négatif. Il faut peut-être, ici aussi, supposer que la polyphonie reçoit dans ce cas une traduction syntaxique: le locuteur, qui présente en arrière-plan une opinion négative, n'adhère évidemment pas à cette opinion : il adopte le point de vue de la proposition affirmative comme celui qui sera vraisemblablement le bon et s'oppose ainsi à l'arrière-plan négatif. Le rapprochement avec un cas particulier de négation explétive pourrait être fécond: les 
questions indirectes du type se demander si...ne pas...pourraient être liées aux constructions à négation explétive du type craindre que ne... Dans les deux cas, la négation pourrait introduire le point de vue selon lequel la proposition nouvelle et non naturellement vraie $\mathrm{P}$ doit être considérée comme fausse aussi longtemps qu'on s'interroge sur elle, tout en étant probable. La contrainte exposée ci-dessus, qui veut que l'interronégative "par hasard» ne soit pas naturellement vraie, vaut également pour craindre que ne (dans ce cas particulier, le «normal » est de plus lié au « souhaitable »; cf. Muller, 1991).

Pour conclure, il me semble que dans ce cas aussi la négation fonctionne comme un jugement de rejet ; s'il y a rejet, il faut qu'il y ait un contenu linguistique en arrière-plan : ce contenu existe bien, mais sous la forme extrêmement vague et générale qu'on a vue, du moins dans le dernier type d'interronégatives examiné. Alors, le lien précis avec l'énoncé est pratiquement rompu : la négation, justifiée par le présupposé global que ce qui n'est pas établi est faux jusqu'à confirmation, n'est plus là que pour être mise en question, et donc pour introduire la proposition comme une assertion presque réalisée. Presque, c'està-dire pas encore.

Wittgenstein, Ludwig, 1980: Philosophische Grammatik, trad. fr.: La grammaire philosophique, Gallimard.

\section{BIBLIOGRAPHIE}

Anscombre, Jean-Claude, et Ducrot Oswald, 1981 : «Interrogation et argumentation », Langue Française, 52.

Attal, Pierre, 1979 : Négationetquantificateurs, doctorat d'Etat, Paris-VIII.

Borillo, Andrée, 1979 : « La négation et l'orientation de la demande de confirmation », Langue Française, 44.

Borillo, Andrée, 1981 : «Quelques aspects de la question rhétorique en français », DRLAV, 25.

Callebaut, Bruno, 1991 : Lanégationenfrançais,uneapprochepragmatico-discursive, Académie royale de Belgique, Bruxelles.

DeCornulier,Benoît, 1985 : Effetsdesens, Ed. de Minuit.

Ducrot, Oswald, 1984 : Ledireetledit, Ed. de Minuit.

Givon, Talmy, 1978: « Negation in Language: Pragmatics, Function, Ontology » in P. Cole, ed. SyntaxandSemantics, 9.

Martin, Robert, 1992 : Pourunelogiquedusens, (2e éd.), PUF.

Moeschler, Jacques, 1991 : « Les aspects pragmatiques de la négation linguistique : acte de langage, argumentation et inférence pragmatique », Travauxducentrede Recherches Sémiologiques, 59, Neuchâtel.

Muller, Claude, 1991 : Lanégationenfrançais, Droz. 
Muller, Claude, 1992 : «La négation comme jugement », LangueFrançaise, 94.

Nølke, Henning, 1992 : « Ne pas : négation descriptive ou polémique? », LangueFrançaise, 94.

Reinhart, Tania, 1986 : « Principes de perception des formes et organisation temporelle des textes narratifs » RecherchesLinguistiquesdeVincennes, 14/15, pp. 45.92.

Searle, John R., 1969 : SpeechActs,Oxford ; trad. fr., 1972 : Lesactesdelangage, Hermann, Paris.

Sennekamp, Marita, 1979 : Die Verwendungsmöglischkeiten von Negationszeichen in Dialogen, Max Hüber V., Tübingen.

\section{NOTES}

1. Selon les règles de Searle, l'acte de négation diffère sur deux points de l'assertion négative, d'après Moeschler : l'une des «conditions préparatoires » est : que p (la proposition sur laquelle porte la négation) « est entretenu dans la mémoire de l'interlocuteur s'il y a négation, ne l'est pas s'il y a assertion négative. L'autre point est la formulation de la condition « essentielle » : pour la négation, « revient à refuser la vérité de $\mathrm{p}$ »; pour l'assertion négative : « revient à assurer que $\mathrm{p}$ représente une situation non réelle». Il faut savoir gré à J. Moeschler d'avoir formulé aussi précisément ces différences: on peut y voir que l'originalité de la négation réside dans l'antériorité d'un contenu propositionnel dont elle dit quelque chose. Par contre, l'assertion négative pose problème. Quelle est la pertinence d'une assertion négative dont le contenu positif n'a pas d'antériorité, et dont la seule justification est de représenter une situation non réelle? Cette catégorie recouvre deux classes bien distinctes d'énoncés, dont l'une n'a pas d'existence linguistique (en tant qu'assertion). Ou bien l'assertion négative dit quelque chose du réel, et alors la négation y est utilisée, comme tout prédicat, pour faire sens en combinaison avec d'autres prédicats - elle est descriptive - ou bien elle représente une situation non réelle, sans aucune justification. De tels énoncés, logiquement vrais, sont en nombre infini à tout moment : si $\mathrm{N}$ est occupé à telle chose, alors je suis en mesure de dire qu'il y a quantité de choses que $\mathrm{N}$ ne fait pas ; si Jean repeint ses volets, alors il n'est pas en train de prendre un bain. La pertinence d'emploi est donc cruciale: ou bien l'énoncé est justifié par une croyance erronée antérieure, qu'il faut éradiquer, ou bien il est descriptivement adéquat, et alors la négation n'est que l'un des éléments descriptifs utilisables : il n'y a pas d'autre possibilité si l'on tient compte de la pertinence dans l'analyse.

2. Pour H. Nølke, 1992, la négation descriptive résulte d'un « effacement » du point de vue sousjacent à la partie positive de l'énoncé, ce qui revient à dire qu'il n'y a plus qu'un point de vue unique associant négation et proposition, celui du locuteur.

3. Les notions d'arrière-plan vs. avant-plan ou premier plan sont parfois utilisées en analyse du discours (cf. T. Reinhart, 1986, pour une application à l'organisation temporelle des récits). Cette terminologie me paraît préférable à celle, logique, de présupposé vs. posé, et d'ailleurs la notion de présupposé, même pragmatique, est impropre à décrire les questions dont le contenu semble anticiper la réponse jugée probable, dans la mesure où le concept de présupposé est lié à l'idée d'antériorité. Quant à la polyphonie, elle distinguera bien des points de vue, mais mal, à mon avis, leur hiérarchisation et leur chronologie. D'autre part, les notions d'arrière-plan et de premier plan peuvent intégrer des éléments contextuels non langagiers sous-jacents (comportement de l'interlocuteur, indices d'erreur) qui justifieront les changements de point de vue à la base de l'énonciation interrogative.

4. Il est clair que divers marqueurs linguistiques sont associés à ces stratégies différentes. Ainsi, on peut paraphraser les questions par anticipation, basées sur le premier plan, par des questions 
en peut-être :

Peut-être ne suis-je pas dans la bonne direction?

5. On devra distinguer: dans la négation "polyphonique » attribuée à l'interlocuteur, il y aura amalgame entre la question $Q$ et la négation NEG :

$[(\mathrm{Q}(\mathrm{NEG}))(\mathrm{P})]$

alors que la question à négation d'anticipation (de premier plan donc) du type de (4), qui autorise au moins une réponse négative, aura pour schéma de portée :

[ Q(NEG (P) ) ]

La prise en compte de la polyphonie semble donc conduire, sinon à des modification dans la hiérarchie des prédicats, du moins à des modifications dans leur association en constituants.

6. Il s'agit alors d'une question d'anticipation, qui «met en scène " le point de vue de premier plan attribué à l'interlocuteur seul. Il me semble difficile de trouver des questions qui, dans le même contexte et avec la même finalité argumentative, utiliseraient l'autre stratégie: jouer la mise en doute attribuée à l'interlocuteur seul d'un AR-PL négatif commun aux interlocuteurs, pour obtenir de lui confirmation de cet AR-PL. De tels énoncés ont plutôt une forme assertée, me semble-t-il :

On ne se dispute jamais, nous deux, n'est-ce pas?

Je ne t'ai jamais laissé tomber, pas vrai?

7. Cet adverbe joue un rôle qu'Andrée Borillo, 1981, souligne à propos des verbes d'opinion. Selon elle, il renforce l'idée d'hypothèse improbable. Ce pourrait être également son sens ici, son usage étant justifié pragmatiquement par le désir du locuteur de se protéger contre une réponse négative en signalant lui-même le caractère fortement hypothétique de la proposition qu'il suggère.

8. Cette analyse doit permettre la distinction entre un arrière-plan négatif réel - présupposé comme on l'a vu - et ce type d'arrière-plan fictif, qui est une mise en perspective sans relation d'antériorité réelle entre l'arrière-plan et le premier plan. La sémantique théorique offre une solution possible, dans ce que R. Martin, 1992, appelle un « univers de croyance »: il distingue l'univers actuel, qui serait celui des AR-PL présupposés pragmatiquement, et l'univers virtuel qui comporte des propositions sur lesquelles le locuteur n'a aucune opinion, mais qui sont «décidables» par lui. Il me semble qu'il y aurait, dans l'univers virtuel, un cas intermédiaire : celui de très nombreuses propositions sur lesquelles le locuteur n'a aucune valeur de vérité à attribuer a priori, mais sur lesquelles il a une opinion - une croyance provisoire, basée sur la plausibilité :

Jusqu'à plus ample informé, normalement : non $P$.

Toute proposition nouvelle conforme à ce présupposé global permettra la création de L'AR-PL fictif négatif.

\section{RÉSUMÉS}

Cet article présente une nouvelle analyse de l'interrogation, basée sur l'opposition entre les connaissances ou présupposés pragmatiques d'arrière-plan et la notion de premier plan, à l'origine de la question. La présence ou non d'une négation peut, dans ce cadre, illustrer la stratégie du locuteur : dans le même contexte, celui-ci peut soit mettre en doute son opinion d'arrière-plan, soit anticiper sur la vérité du premier plan. On peut ainsi décrire l'interronégative sans faire intervenir la rhétorique de l'argumentation et du dialogue, également exprimable dans 
ce cadre avec les distinctions polyphoniques d'énonciateurs.

Cette analyse conduit à la mise en évidence d'un type surprenant de question: les interronégatives en "par hasard ", dont on montre que l'origine probable est à chercher dans un arrière-plan faisant partie des savoirs partagés : tout élément non avéré est a priori concevable comme un présupposé négatif - à l'exception notable d'états de faits généralement posés comme positifs, et qui précisément bloquent la formation de ce type de questions.

This paper proposes a new analysis of the interrogatives, based on the opposition between knowledges or pragmatic presuppositions from the background, and the foreground which is originating the question. The presence or absence of a negation can in this frame illustrate the internal strategy of the speaker: in the same context, he can either express a doubt about his background belief, or anticipate the probable foreground truth. We thus can describe the interronegatives without the necessity of having recourse to the external notions of dialogic strategies or conversational rhetoric - although the rhetoric questions could also be treated in the same way.

This analysis put forth the peculiarities of a surprising type of questions: the interronegatives of the " par hasard» type, whose probable origin lies in a general backgroung: any unknown (thus unconfirmed) event is expressible as a negative presupposition. The noticeable exceptions are general positive beliefs, which precisely do not permit this type of questions.

\section{AUTEUR}

\section{CLAUDE MULLER}

Bordeaux III 